\title{
Interpolation Environment of Tensor Mathematics at the Corpuscular Stage of Computational Experiments in Hydromechanics
}

\author{
Alexander Bogdanov ${ }^{1, \star}$, Alexander Degtyarev ${ }^{1, \star \star}$, Vasily Khramushin ${ }^{1, \star \star \star}$, and \\ Yulia Shichkina ${ }^{1,2, \star \star \star \star}$ \\ ${ }^{1}$ St. Petersburg State University, Universitetskii prospect 35,198504 Saint-Petersburg, Russia \\ ${ }^{2}$ St. Petersburg State Electrotechnical University, Popova ulitca 5, 197376 Saint-Petersburg, Russia
}

\begin{abstract}
Stages of direct computational experiments in hydromechanics based on tensor mathematics tools are represented by conditionally independent mathematical models for calculations separation in accordance with physical processes. Continual stage of numerical modeling is constructed on a small time interval in a stationary grid space. Here coordination of continuity conditions and energy conservation is carried out. Then, at the subsequent corpuscular stage of the computational experiment, kinematic parameters of mass centers and surface stresses at the boundaries of the grid cells are used in modeling of free unsteady motions of volume cells that are considered as independent particles. These particles can be subject to vortex and discontinuous interactions, when restructuring of free boundaries and internal rheological states has place. Transition from one stage to another is provided by interpolation operations of tensor mathematics. Such interpolation environment formalizes the use of physical laws for mechanics of continuous media modeling, provides control of rheological state and conditions for existence of discontinuous solutions: rigid and free boundaries, vortex layers, their turbulent or empirical generalizations.
\end{abstract}

\section{Introduction}

The paper discusses some generalizations and justifications for the practical application of continualcorpuscular models of the computational hydromechanics. Tensor mathematics in the simulation of processes in continuous medium mechanics synthesizes two-stage computational experiments with the help of the principal (linear) components of the hydromechanics laws in the vicinity of adjacent fluid particles at controlled time intervals [1]. The use of the classical apparatus of the tensor calculus allows one to formalize the key approximations of hydromechanics in the three-dimensional space and the absolute time. This creates the necessary tools and ample mechanisms for direct algorithmic modeling of various spatio-temporal processes in the nearest environment of a computational cell.

\footnotetext{
^e-mail: a.v.bogdanov@spbu.ru

$\star \star$ e-mail: a.degtyarev@spbu.ru

$\star \star \star$ e-mail: v.khram@gmail.com

$\star \star \star \star$ e-mail: strange.y@mail.ru
} 
An intuitive-natural variant of a direct computational experiment constructing is represented by two stages of spatial integration of the first order. These stages connect the differentials of kinematic and rheological processes into the general solution of mechanics equations by means of extrapolation of the second order in time.

At the first, continual stage, the traditional kinematic increments (differentials) within the original numerical cells-corpuscles are determined. These are accelerations in the motion-rotationdeformation arising under the action of volume / mass forces and surface stresses on the boundaries between adjacent nodes of elementary numerical cells.

At the second, corpuscular stage, the updated, nonstationary physical processes are constructed over a number of formally independent corpuscular particles, the mechanics of interaction of which directly affects the change in the rheological properties of the continuous medium. After this, the local surface stresses are rebuilt in the original nodes of the grid calculation area.

Then the essence of the computational experiment is reduced to the following [2, 3]. (1) Free polarized corpuscles in the initial stress field accelerate the kinematic flows of a continuous medium. There is a substitution of the emerging depressions by the anisotropic material substance (a functionally generalized physical field). (2) At the corpuscular stage of the calculations, physical fields are updated in empirical gradient, direct linear and divergent dependences for volumetric and surface stresses on liquid particles, taking into account the current rheological state of the spatial flows of the continuous medium.

The flows and transformation of the material medium are uniformly formalized in tensor form on the continuum and corpuscular stages of the computational experiment.

\section{Direct continual-corpuscular computational experiment}

A computational experiment in hydromechanics is designed on the basis of explicit schemes on two stages of physical processes modeling and 3D tensor mathematics. A specially synthesized functionalalgorithmic apparatus with a strict and definite representation of mechanics and physics laws in architecture and programming languages of modern computers is used [4].

Additive growth of physical quantities occurs according to a single scalar argument in time. All spatial operations are based on successive products defining mechanics laws as interpolation dependencies between the internal Cartesian and transformed Euclidean bases of the rheological characteristics of the continuous medium. The syntax of the order of multiplicands is the following:

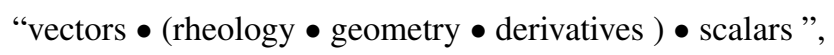

where rheological and geometric tensors always exist, while derivatives (local velocities and strains) can be degenerate. Vector quantities are used in the reconstruction of physical and geometric bases in mechanics laws. Scalars are invariant under spatial transformations. In particular, they are used in the traditional differentiation / integration of hydromechanical equations in time.

Correct work with information about current state of simulated continuous medium and controlled execution of mechanics laws are provided by the following key rules of tensor mathematics:

I - numerical objects are defined in dimensional form;

II - all computational operations are context-sensitive, i.e. they have first order approximation in space and time;

III - duality of absolute and local reference frames, i.e. separation into stages of conditionally independent physical processes.

In what follows, we use uppercase letters for physical quantities in the global reference frame, and lowercase for quantities in connected local bases, which are essentially difference differentials. Notations are described in details in $[5,6]$. 
Now we describe the mathematical and algorithmic features of the build up of a computational experiment.

1. In the computational experiment, the first continual or kinematic stage is built on the initial nodes of the grid region according to the specified mass inertial forces and stresses on the conjugate faces of the computational cells. The tensor models of the rheological state of the simulated fluid participate in multiplications. These models include the prehistory of the hydrodynamic processes for relaxation matching according to the "forecast and correction" scheme in the equations of motion. The result of the calculations is the updated space of increments in the intensity of the continuous medium flows. It fixes the displacements of the mass centers of the numerical cells and the current convective velocities in conjugate nodes of the computational domain.

The generalized laws of the mechanics apply to smooth spatial functions: momentum vectors $\vec{v} \cdot \mathbf{m}$ $=\vec{f} \cdot t[\mathrm{~kg} \cdot \mathrm{m} / \mathrm{s}]$ and tensors of intensities of the internal flows in the tensor form $\stackrel{v}{\boldsymbol{\rho}} \cdot \hat{\mathbf{v}}=\stackrel{\vee}{\mathbf{r}} \cdot \hat{\mathbf{f}} \cdot t=\overrightarrow{\mathbf{f}} \cdot t$ $[\mathrm{kg} / \mathrm{s}]$.

2. At the second corpuscular stage the mechanics of the interaction of the virtual numerical objects which inherit the specific physical (rheological) properties of the continuous medium is considered:

Elasticity $\rightarrow$ displacement - cell compression;

Viscosity $\rightarrow$ radiation - diffusion rate;

Inertia $\rightarrow$ flow shift - acceleration of corpuscles.

The reconstruction of the kinematics of the flows affects the distribution of the mass forces and surface stresses. Kinematic displacements of mobile and deformed liquid particles are added to harmonize the laws of hydromechanics and rheology of the internal state of the particles at the corpuscular stage. An algorithmic sequence is formed: \{new synthesized bases $\rightarrow$ local and convective velocities $\rightarrow$ stresses $\}$

At the corpuscular stage, the calculation nodes are shifted to the centers of the virtual numeric cells. This requires algorithmic methods for quick selection of values in conjugate interpolation spaces $\left\{{ }^{\circ} \hat{\mathbf{r}}={ }_{+}^{\circ} \vec{R}_{i}-{ }_{\circ}^{\circ} \vec{R}_{i}\right\}\left[\mathrm{m}^{3}\right]$, where new coordinates of the centers of mass are reconstructed using the differences $\left\{{ }^{+} \hat{\mathbf{r}}={ }_{+}^{+} \vec{R}_{i}-{ }_{\circ}^{\circ} \vec{R}_{i}\right\}\left[\mathrm{m}^{3}\right]$.

In the case of small deformations inherent in models of the theory of elasticity, the local displacement tensor is formed by summation over the entire time period. In fluid mechanics, the local velocity and displacement $\left\{\hat{\mathbf{v}} \cdot t={ }^{+} \hat{\mathbf{r}}-{ }^{\circ} \hat{\mathbf{r}}\right\}\left[\mathrm{m}^{3}\right]$ are supplemented by velocity (acceleration) increments of the calculated time interval $t$ [s]. They are calculated using the updated velocities of the numeric cells $\left\{{ }^{+} \hat{\omega}={ }_{+}^{+} \vec{V}_{i}-{ }_{\circ}^{\circ} \vec{V}_{i}\right\}\left[\mathrm{m}^{3} / \mathrm{s}\right]$, or they are selected from the first stage in the case of small deformations $\hat{\omega}=\vec{\omega}^{i}=\Delta \vec{V}_{i}=_{+} \vec{V}_{i}-\circ \vec{V}_{i}\left[\mathrm{~m}^{3} / \mathrm{s}\right]$.

The rheology of the continuous environment is proved in commensurable scales. It is formalized by simple expressions: $\left\{{ }^{+} \mathbf{m}={ }^{\circ} \mathbf{m}+f(\stackrel{\vee}{\boldsymbol{\rho}} \cdot \hat{\mathbf{v}}) \cdot t\right\}[\mathrm{kg}]$, where $\mathbf{m}[\mathrm{kg}]$ is the inertial mass of the liquid particle; $f(\stackrel{\vee}{\rho} \cdot \hat{\mathbf{v}})[\mathrm{kg} / \mathrm{s}]$ is actually the law of mechanics as tensor function of the product of internal state (density of liquid particle $\left.\stackrel{\vee}{\rho}\left[\mathrm{kg} / \mathrm{m}^{3}\right)\right]$ ) under the influence of the local speeds tensor $\hat{\mathbf{v}}\left[\mathrm{m}^{3} / \mathrm{s}\right]$.

In the space of the trivectors of the local velocities, deformation displacements relative to moving corpuscles are specified: $\hat{\boldsymbol{\varepsilon}} \approx \hat{\mathbf{v}} \cdot t\left[\mathrm{~m}^{3}\right]$. Also the tensor of tension is determined: $\stackrel{<}{\boldsymbol{\sigma}}=(\hat{\boldsymbol{\varepsilon}} \cdot \boldsymbol{\kappa}+\hat{\boldsymbol{\omega}} \cdot \hat{\boldsymbol{\eta}}) \times \lambda$ $\left[\mathrm{kg} \mathrm{m} / \mathrm{c}^{2}\right]$, where $\boldsymbol{\kappa}\left[\mathrm{N} / \mathrm{m}^{3}\right]$ is the modulus of elastic deformation; $\stackrel{\vee}{\boldsymbol{\eta}}\left[\mathrm{N} \mathrm{s} / \mathrm{m}^{3}\right]$ is the tensor of speed viscosity; $\lambda[\mathrm{m}]$ is the scale coefficient characterizing the distance between the interacting liquid particles.

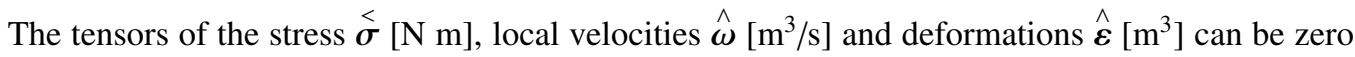
or degenerate. It is excluded the calculation of their inversions. Change of bases is performed only in 
operations of product with the form tensors, such as $\overrightarrow{\mathbf{v}}=\stackrel{\vee}{\mathbf{r}} \cdot \hat{\mathbf{v}}[1 / \mathrm{s}]$ or $\stackrel{\mathbf{v}}{\mathbf{v}} \hat{\mathbf{r}} \cdot \stackrel{\vee}{\mathbf{v}}[1 / \mathrm{s}]$ without affecting the time count in the denominator.

\section{Conclusion}

The described procedure of the computational experiment takes into account all rheological properties of the medium. The constructed scheme ensures unambiguous interpretation of physical processes by means of linear interpolation, which is performed by tensor operators in conjugate coordinate systems [5]. The solution is carried out in three stages:

1. integral accumulation of the amount of motion and deformation of particles of liquid;

2. local displacements and deformations of liquid particles over the calculated time step;

3. increment of velocities for local displacements and deformations of virtual numerical cells.

The continuum (Eulerian) and corpuscular (Lagrange) stages are connected by the iterative matching of the kinematic and rheological characteristics of the physical field in explicit numerical schemes. In this situation, we obtain non-stationary computational processes according to mathematical models for viscous, elastic and compressible liquids.

Scalar temperature distribution, variation of elastic gas density related with sound velocity, and other characteristics can be taken into account in applied problems in the calculation of internal flows of moving particles. Due to the use of explicit numerical schemes, it is possible to introduce empirical refinements during calculations. This synthesizes an extended solution of the applied problem of aerodynamic mechanics. In such problems tensor spaces are represented by linear interpolation dependencies in space and time. This applies to smooth distributions of scalar, vector and tensor anisotropic spatial functions. Such functions are valid in the kinematic calculations of the interaction of particles, when we have similar stages in the synthesis of vector gradients and the analysis of resulting divergences for local density changes. In the tensor formulation, the modeling algorithms correspond to the properties of real liquids. Virtual computing processes are interpreted by inertial external and internal interactions of particle-cells.

\section{Acknowledgement}

The research is partly supported by Russian Foundation for Basic Research (project N 16-07-00886, 16-07-01113, 17-29-04288). The paper has been prepared within the scope of the state project "Initiative scientific project" of the main part of the state plan of the Ministry of Education and Science of Russian Federation (task N 2.6553.2017/8.9 BCH Basic Part).

\section{References}

[1] A. B. Degtyarev and V. N. Khramushin, Mathematical Modeling 26, 11, 4-17 (2014)

[2] A. Bogdanov, A. Degtyarev, and V. Korkhov, EPJ Web of Conferences 108, 01002 (2016)

[3] A. Bogdanov and V. Khramushin, EPJ Web of Conferences 108, 02013 (2016)

[4] A. Degtyarev, V. Khramushin, and Y. Shichkina, AIP Conference Proceedings 1863, 110006 (2017)

[5] A. Degtyarev and V. Khramushin, EPJ Web of Conferences 108, 02018 (2016)

[6] V. N. Khramushin, 3D Tensor Mathematics for the Computational Fluid Mechanics Experience (FEB RAS, Vladivostok, 2005) 